\title{
Late-Onset Orbital Cellulitis with Abscess Formation Caused by Klebsiella Pneumoniae*
}

\author{
Jing Li, Jianmin Ma", Xin Ge \\ Beijing Tongren Eye Center, Beijing Ophthalmology and Visual Sciences Key Laboratory, Beijing Tongren Hospital, Capital Medical \\ University, Beijing, China. \\ Email: \#mma@sina.com
}

Received June $27^{\text {th }}, 2012$; revised July $30^{\text {th }}, 2012$; accepted August $15^{\text {th }}, 2012$

\begin{abstract}
Klebsiella pneumoniae is a facultative anaerobic gram-negative, non-motile, capsulated, gas-producing rod found widely in nature and often associated with urinary and respiratory infections in humans. Orbital cellulitis with abscess formation caused by K. pneumoniae is rare. Here, we present a case of $\mathrm{K}$. pneumonia-inducing orbital cellulitis with abscess formation in a patient who had undergone an orbital wall fracture prosthesis with hydroxyapatite implantation due to orbital trauma 9 years ago. The patient was treated successfully with antibiotics and surgery.
\end{abstract}

Keywords: Orbital Cellulitis; Abscess; Klebsiella Pneumoniae

\section{Case Report}

A 52-year-old man presented to our ophthalmology department with a 1 week history of eye pain, blepharedema, conjunctival congestion, and proptosis in the left eye. 7 days prior, he had suddenly developed a fever of $39.0^{\circ} \mathrm{C}$ after a continuous period of being overworked. A tentative diagnosis of orbital cellulitis was made and the patient was treated with intravenous cefoxitin sodium as an inpatient. Most of the symptoms slightly improved. Nine years before his presentation, the patient had been admitted to our hospital for the first time with orbital trauma following a traffic accident. He had received an orbital wall fracture prosthesis with hydroxyapatite implantation. He denied recent facial trauma or dental work. Visual acuity was $20 / 20$ in both eyes. The adduction of the left eye was severely restricted. Due to his orbital trauma and surgical history, we advised him to undergo an orbital computed tomography. To our surprise, a huge abscess formation was seen adjacent to the implant of the medial wall of the left orbit (Figure 1). The laboratory findings showed a high level of leukocytes, erythrocyte sedimentation rate, and C-reactive protein. Under general anesthesia, incision and drainage of the orbital abscess was performed. The purulent material was collected and cultured immediately. The isolate was found to be gramnegative. Using the Vitek II automated system (bioMérieux,

\footnotetext{
"Conflict of Interest: None of the authors has conflict of interest with the submission. Financial support: No financial support was received for this submission.

"Corresponding author.
}

Marcyl'Etoile, France), the isolate was confirmed as extended-spectrum $\beta$-lactamase-producing Klebsiella pneumoniae, with a $96 \%$ confidence level. Antimicrobial susceptibility test was performed using the Vitek II automated system which resulted in the MICs shown in Table 1. The Kirby-Bauer agar diffusion method was used as a confirmatory tool. The Clinical and Laboratory Standards Institute (CLSI) guidelines for susceptibility testing of K. pneumoniae were followed. After surgery, the patient was managed with intravenous piperacillin/tazobactam $4.5 \mathrm{mg} / 12 \mathrm{~h}$ for 7 days and imipenem/cilastatin sodium $250 \mathrm{mg} / 12 \mathrm{~h}$ for 14 days. His symptoms subsided completely and laboratory findings became normal without recurrence for 6 months.

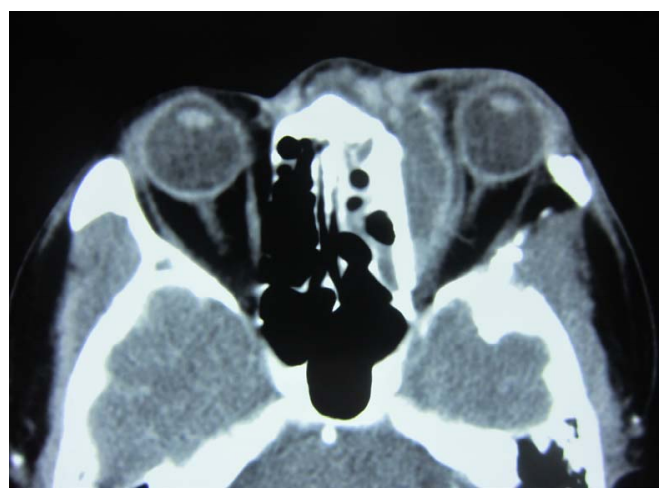

Figure 1. Computed tomography of the orbit showing orbital cellulitis with a huge abscess adjacent to the hydroxyapatite implant of the medial wall of the left orbit and partial pneumatization of the left ethmoidal sinus. 
Table 1. Disk susceptibilities of the $\mathrm{K}$. pneumoniae isolates.

\begin{tabular}{lcc}
\hline \multicolumn{1}{c}{ Antibiotic } & MIC $(\mu \mathrm{g} / \mathrm{ml})$ & Susceptibility $^{\alpha}$ \\
\hline Ampicillin & $\geq 32$ & $\mathrm{R}$ \\
Ampicillin/sulbactam & 16 & $\mathrm{I}$ \\
Piperacillin/tazobactam & 8 & $\mathrm{~S}$ \\
Cefazolin & $\leq 4$ & $\mathrm{R}$ \\
Cefotetan & $\leq 4$ & $\mathrm{~S}$ \\
Ceftazidime & $\leq 1$ & $\mathrm{~S}$ \\
Ceftriaxone & $\leq 1$ & $\mathrm{~S}$ \\
Cefepime & $\leq 1$ & $\mathrm{~S}$ \\
Aztreonam & $\leq 1$ & $\mathrm{~S}$ \\
Ertapenem & 0.25 & $\mathrm{~S}$ \\
Imipenem & $\leq 1$ & $\mathrm{~S}$ \\
Amikacin & $\leq 2$ & $\mathrm{~S}$ \\
Gentamicin & $\leq 1$ & $\mathrm{~S}$ \\
Tobramycin & $\leq 1$ & $\mathrm{~S}$ \\
Ciproxacin & 1 & $\mathrm{~S}$ \\
Levofloxacin & 1 & $\mathrm{~S}$ \\
Nitrofurantoin & 256 & $\mathrm{R}$ \\
Trimethoprim/sulfanilamide & $2 / 38$ & $\mathrm{~S}$ \\
\hline R & &
\end{tabular}

${ }^{\alpha} \mathrm{R}$, resistant; I, intermediary; S, susceptible

\section{Discussion}

K. pneumoniae is a facultative anaerobic gram-negative, non-motile, capsulated, gas-producing rod found commonly in nature and is often associated with urinary and respiratory infections in humans. K. pneumoniae is the most common and most important rod of the Klebsiella species that are identified as pathogenic to humans and include K. pneumoniae, R. oxytoca, K. ornithinolytica, K. planticola, and $\mathrm{K}$. terrigena. It is also a common opportunistic pathogen in humans; therefore, immunocompromised and/or postoperative patients are prone to suffer from infections with this pathogen. In addition, K. pneumoniae is one of the most important pathogen that can product extended-spectrum beta-lactamases, leading to its resistance to most antibiotics.

$\mathrm{K}$. pneumoniae is associated with wound infections [1$3]$, respiratory tract infections [4], arthritis [5-8], osteomyelitis [5,7], septicemia [5,9], meningitis [5,10-11], genitourinary infection [7,12], and so on. Eyeball infections due to K. pneumoniae is not rare and can cause keratitis [13], conjunctivitis [14] and endophthalmitis [1517]. However, to the best of our knowledge, orbital cellulitis caused by K. pneumoniae has rarely been reported in the literature [18-20].

Orbital cellulitis and abscess are medical emergencies.
Delayed or inadequate management may lead to permanent loss of vision [21-23]. Therefore, history-taking, clinical examination, and laboratory investigation including proper orbital imaging is essential in determining the source of infection [24]. There are various sources of orbital cellulitis, including the sinuses, open fractures, foreign bodies, periocular surgery, Munchausen's syndrome, intraocular tumors, and so on $[24,25]$. The skin and the sinuses are colonized by various microorganisms. Orbital cellulitis following trauma is due to direct exposure of the orbital contents to these microorganisms [24]. Open and closed periorbital fractures involving the sinuses or the nasal bone, may be a risk factor for orbital infection [26,27]. The most common bacteria isolated from pediatric and adult patients with community-acquired acute purulent sinusitis are Streptococcus pneumoniae, Haemophilus influenzae, Moraxella catarrhalis, and Streptococcus pyogenes. Staphylococcus aureus and anaerobic bacteria (Prevotella and Porphyromonas, Fusobacterium and Peptostreptococcus spp) are the main isolates in chronic sinusitis [28]. Infection may present within a few hours to several years after trauma [23,24].

After some surgeries involving the orbit, there is an increased risk for infection, especially in the presence of alloplastic foreign bodies. Cataract surgery $[29,30]$, blepharoplasty [31], strabismus surgery [32,33], and other 
procedures may all expose the orbit to infection $[34,35]$. Therefore, patients undergoing any interventional procedures should be closely monitored for signs of infection and should be treated aggressively if evidence of an infection is found [24].

Because K. pneumoniae is almost extended-spectrum $\beta$-lactamase-producing bacillus, it is resistant to most antibiotics. Susceptibility testing is necessary to help physicians choose the appropriate antibiotics in clinic. In our case, the results of the susceptibility testing showed that piperacillin/tazobactam, cefazolin, cefotetan, ceftazidime, ceftriaxone, cefepime, aztreonam, ertapenem, imipenem, amikacin, gentamicin, tobramycin, ciproxacin, levofloxacin are all effective. However, the extended-spectrum $\beta$-lactamase-producing bacteria, which show susceptible to penicillins, cephalosporins and aztreonam often become resistant to them. Therefore, $\beta$-lactamase inhibitors and carbapenems are recommended in clinical practice. Our patient was treated with intravenous piperacillin/ tazobactam for 7 days and oral imipenem for 14 days after surgery without recurrence for 6 months.

\section{Conclusion}

To our knowledge, this is the first case of late-onset orbital cellulitis and abscess formation induced by K. pneumoniae after an orbital wall fracture prosthesis with hydroxyapatite implantation reported in the literature. Orbital cellulitis and abscess formation due to K. pneumoniae should be considered, whenever patients suffer the orbital trauma and undergo surgery.

\section{REFERENCES}

[1] E. Rezaei, H. Safari, M. Naderinasab, et al., "Common Pathogens in Burn Wound and Changes in Their Drug Sensitivity," Burns, Vol. 37, No. 5, 2011, pp. 804-806. doi:10.1016/j.burns.2011.01.019

[2] M. I. Ahmed, "Prevalence of Nosocomial Wound Infection among Postoperative Patients and Antibiotics Patterns at Teaching Hospital in Sudan," North American Journal of Medical Sciences, Vol. 4, No. 1, 2012, pp. 2934. doi:10.4103/1947-2714.92900

[3] A. K. Seth, M. R. Geringer, A. N. Gurjala, et al., "Understanding the Host Inflammatory Response to Wound Infection: An in Vivo Study of Klebsiella Pneumoniae in a Rabbit Ear Wound Model," Wound Repair and Regeneration, Vol. 20, No. 2, 2012, pp. 214-225. doi:10.1111/j.1524-475X.2012.00764.x

[4] S. Ghafourian, Z. B. Sekawi, N. Sadeghifard, et al., "The prevalence of ESBLs Producing Klebsiella Pneumoniae Isolates in Some Major Hospitals, Iran," Open Microbiology Journal, Vol. 5, 2011, pp. 91-95. doi:10.2174/1874285801105010091

[5] Z. Ghorashi, N. Nezami, H. Hoseinpour-Feizi, et al., "Arthritis, Osteomyelitis, Septicemia and Meningitis Caused by Klebsiella in a Low-Birth-Weight Newborn: A Ca- se Report," Journal of Medical Case Reports, Vol. 5, 2011, p. 241. doi:10.1186/1752-1947-5-241

[6] S. Schelenz, K. Bramham and D. Goldsmith, "Septic Arthritis Due to Extended Spectrum Beta Lactamase Producing Klebsiella Pneumoniae," Joint Bone Spine, Vol. 74 , No. 3, 2007, pp. 275-278.

doi:10.1016/j.jbspin.2006.08.007

[7] L. C. Chew, "Septic Monoarthritis and Osteomyelitis in an Elderly Man Following Klebsiella Pneumoniae Genitourinary Infection: Case Report," Annals of the Academy of Medicine Singapore, Vol. 35, No. 2, 2006, pp. 100103.

[8] J. E. Kohler, M. P. Hutchens, P. M. Sadow, et al., "Klebsiella Pneumoniae Necrotizing Fasciitis and Septic Arthritis: An Appearance in the Western Hemisphere," Surgical Infections, Vol. 8, No. 2, 2007, pp. 227-232. doi:10.1089/sur.2006.007

[9] N. Y. Lee, W. H. Huang, K. C. Tsui, et al., "Carbapenem Therapy for Bacteremia Due to Extended-Spectrum (Beta)-Lactamase-Producing Escherichia Coli or Klebsiella Pneumoniae," Diagnostic Microbiology and Infectious Disease, Vol. 70, No. 1, 2011, pp. 150-153.

doi:10.1016/j.diagmicrobio.2010.12.008

[10] A. Stucki, M. Cottagnoud, F. Acosta, et al., "Efficacy of Doripenem against Escherichia Coli and Klebsiella Pneumoniae in Experimental Meningitis," Journal of Antimicrobial Chemotherapy, Vol. 67, No. 3, 2012, pp. 661665. doi:10.1093/jac/dkr482

[11] W. N. Chang, C. H. Lu, C. R. Huang, et al., "Clinical Characteristics of Post-Neurosurgical Klebsiella Pneumoniae Meningitis in Adults and a Clinical Comparison to the Spontaneous Form in a Taiwanese Population," Journal of Clinical Neuroscience, Vol. 17, No. 3, 2010, pp. 334-338. doi:10.1016/j.jocn.2009.06.019

[12] D. A. Rosen, J. S. Pinkner, J. N. Walker, et al., "Molecular Variations in Klebsiella Pneumoniae and Escherichia coli FimH Affect Function and Pathogenesis in the Urinary Tract," Infection and Immunity, Vol. 76, No. 7, 2008, pp. 3346-3356. doi:10.1128/IAI.00340-08

[13] S. Zarei-Ghanavati, M. R. Sedaghat and A. GhavamiShahri, "Acute Klebsiella Pneumoniae Interface Keratitis after Deep Anterior Lamellar Keratoplasty," Japanese Journal of Ophthalmology, Vol. 55, No. 1, 2011, pp. 7476. doi:10.1007/s10384-010-0836-7

[14] T. Aung and T. K. Chan, "Nosocomial Klebsiella Pneumoniae Conjunctivitis Resulting in Infectious Keratitis and Bilateral Corneal Perforation," Cornea, Vol. 17, No. 5, 1998, pp. 558-561. doi:10.1097/00003226-199809000-00015

[15] A. H. Kashani and D. Eliott, "Bilateral Klebsiella Pneumoniae (K1 Serotype) Endogenous Endophthalmitis as the Presenting Sign of Disseminated Infection," Ophthalmic Surgery, Lasers \& Imaging, Vol. 42, 2011, pp. e12e14.

[16] L. P. Ang, H. M. Lee, K. G. Au Eong, et al., "Endogenous Klebsiella Endophthalmitis," Eye, Vol. 14, No. 6, 2000, pp. 855-860. doi:10.1038/eye.2000.236

[17] Y. J. Chen, H. K. Kuo, P. C. Wu, et al., "A 10-Year Comparison of Endogenous Endophthalmitis Outcomes: An 
East Asian Experience with Klebsiella Pneumoniae Infection," Retina, Vol. 24, No. 3, 2004, pp. 383-390. doi:10.1097/00006982-200406000-00008

[18] S. J. Yang, S. Y. Park, Y. J. Lee, et al., "Klebsiella Pneumoniae Orbital Cellulitis with Extensive Vascular Occlusions in a Patient with Type 2 Diabetes," Korean Journal of Internal Medicine, Vol. 25, No. 1, 2010, pp. 114-117. doi:10.3904/kjim.2010.25.1.114

[19] I. A. Chaudhry, F. A. Shamsi, E. Elzaridi, et al., "Inpatient Preseptal Cellulitis: Experience from a Tertiary Eye Care Centre," British Journal of Ophthalmology, Vol. 92, No. 10, 2008, pp. 1337-1341. doi:10.1136/bjo.2007.128975

[20] C. T. Lin and Y.Y. Tsai, "Klebsiella Pneumoniae Orbital Cellulitis," Chinese Medical Journal (Taipei), Vol. 64, No. 9, 2001, pp. 551-554.

[21] A. Hauser and S. Fogarasi, "Periorbital and Orbital Cellulitis," Pediatrics in Review, Vol. 31, No. 6, 2010, pp. 242249.

[22] M. Coskun, Ö. IIhan, U. Keskin, et al., "Central Retinal Artery Occlusion Secondary to Orbital Cellulitis and Abscess Following Dacryocystitis," European Journal of Ophthalmology, Vol. 21, No. 5, 2011, pp. 649-652. doi:10.5301/EJO.2011.6493

[23] K. Starska, M. Lukomski and D. Starska-Dawidowskda, "Preseptal Orbital Abscess as a Post-Traumatic Late Complication-Review of the Literature and Case Report," Otolaryngologia Polska, the Polish Otolaryngology, Vol. 61, No. 3, 2007, pp. 331-334.

[24] S. Rumelt and P. A. Rubin, "Potential Sources for Orbital Cellulitis," International Ophthalmology Clinics, Vol. 36, No. 3, 1996, pp. 207-221. doi:10.1097/00004397-199603630-00019

[25] P. Laloyaux, D. Vanpee and J. B. Gillet, “Orbital Cellulitis with Abscess Formation Caused by Frontal Sinusitis," Journal of Emergency Medicine, Vol. 18, No. 2, 2000, pp. 253-254. doi:10.1016/S0736-4679(99)00204-8

[26] G. J. Ben Simon, S. Bush, D. Selva, et al., "Orbital Cellulitis: A Rare Complication after Orbital Blowout Fracture," Ophthalmology, Vol. 112, No. 11, 2005, pp. 20302034. doi:10.1016/j.ophtha.2005.06.012
[27] S. P. Bord and J. Linden, "Trauma to the Globe and Orbit," Emergency Medicine Clinics of North America, Vol. 26, No. 1, 2008, pp. 97-123. doi:10.1016/j.emc.2007.11.006

[28] I. Brook, "Microbiology of Sinusitis," Proceedings of the American Thoracic Society, Vol. 8, No. 1, 2011, pp. 90100. doi:10.1513/pats.201006-038RN

[29] V. Kumar and D. Saunders, "Orbital Cellulitis after Phacoemulsification and Intraocular Lens Implantation," Journal of Cataract and Refractive Surgery, Vol. 30, No. 4, 2004, pp. 918-920. doi:10.1016/j.jcrs.2003.11.026

[30] S. Y. Liang, G. Moloney, B. A. O’Donnell, et al., “Orbital Cellulitis as a Postoperative Complication of SubTenon Anaesthesia in Cataract Surgery," Clinical and Experimental Ophthalmology, Vol. 34, No. 9, 2006, pp. 897-899. doi:10.1111/j.1442-9071.2006.01361.x

[31] V. Juthani, C. I. Zoumalan, R. D. Lisman, et al., "Successful Management of Methicillin-Resistant Staphylococcus aureus Orbital Cellulitis after Blepharoplasty," Plastic and Reconstructive Surgery, Vol. 126, No. 6, 2010, pp. 305e-307e. doi:10.1097/PRS.0b013e3181f63f67

[32] E. Hoyama, V. Limawararut, I, Leibovitch, et al., "Blinding Orbital Cellulitis: A Complication of Strabismus Surgery," Ophthalmic Plastic and Reconstructive Surgery, Vol. 22, No. 6, 2006, pp. 472-473. doi:10.1097/01.iop.0000245486.61228.8d

[33] A. Basheikh and R. Superstein, "A Child with Bilateral Orbital Cellulitis One Day after Strabismus Surgery," Journal of AAPOS, Vol. 13, No. 5, 2009, pp. 488-490. doi:10.1016/j.jaapos.2009.05.016

[34] M. P. Hatton and M. L. Durand, "Orbital Cellulitis with Abscess Formation Following Surgical Treatment of Canaliculitis," Ophthalmic Plastic and Reconstructive Surgery, Vol. 24, No. 4, 2008, pp. 314-316. doi:10.1097/IOP.0b013e31817e9be7

[35] M. M. Marcet, J. J. Woog, A. R. Bellows, et al., "Orbital Complications after Aqueous Drainage Device Procedures," Ophthalmic Plastic and Reconstructive Surgery, Vol. 21, No. 1, 2005, pp. 67-69. doi:10.1097/01.IOP.0000150351.91768.16 